\title{
Retraction notice for: "Ginsenoside Rd inhibits IL-1ß- induced inflammation and degradation of intervertebral disc chondrocytes by increasing IL1RAP ubiquitination" [Braz J Med Biol Res (2019) 52(9): e8525]
}

\author{
Wei-Jia Zhang (ii) ${ }^{1}$, Ying Liu (ii) ${ }^{1}$, Jie-Shu Wei(i) ${ }^{1}$, and Ya-Li Wu (i) ${ }^{2}$
}

${ }^{1}$ School of Pharmacy, Xinhua College of Sun Yat-Sen University, Tianhe District, Guangzhou City, Guangzhou, China ${ }^{2}$ School of Rehabilitation Medicine, Xinhua College of Sun Yat-sen University, Tianhe District, Guangzhou City, Guangzhou, China

Retraction for: Braz J Med Biol Res | doi: 10.1590/1414-431x20198525 | PMCID: PMC6694592 | PMID: 31411316

The authors would like to retract the article "Ginsenoside Rd inhibits IL-1 $\beta$-induced inflammation and degradation of intervertebral disc chondrocytes by increasing IL1RAP ubiquitination" that was published in volume 52 no. 9 (2019) (Epub Aug 12, 2019) in the Brazilian Journal of Medical and Biological Research <http://dx.doi.org/10.1590/1414-431x2019 8525 > PMCID: PMC6694592 | PMID: 31411316.

The Corresponding author Ya-Li Wu states that "there is a conflict between authors concerning the publication of Figure $1 \mathrm{Aa}$ and $\mathrm{Ab}$, which had already been published in a Chinese Journal". Therefore, this article is being retracted and all authors will be prohibited to publish in the Brazilian Journal of Medical and Biological Research in the future. 